\title{
ACRYLAMIDE FORMATION DURING THE FRYING OF BEEF BURGER: EFFECT OF TEMPERATURE AND TIME
}

\author{
Samaneh Ghasemian ${ }^{1}$, Karamatollah Rezaei ${ }^{*, 2}$, Reza Abedini**,3 ${ }^{*}$ Hashem Poorazarang ${ }^{1}$ \\ ${ }^{1}$ Department of Food Science and Technology, Faculty of Agricultural Engineering, Ferdowsi University of Mashhad, Mashhad, Iran \\ ${ }^{2}$ Department of Food Science, Engineering and Technology, Faculty of Agricultural Engineering, University of Tehran, Karaj, Iran \\ ${ }^{3}$ Department of Chemical Engineering, Faculty of Chemical Engineering, Tarbiat Modares University, P.O. Box 14115-143, Tehran, Iran
}

Received 15 May 2011; accepted 11 September 2011

\begin{abstract}
Acrylamide $\left(\mathrm{CH}_{2}=\mathrm{CHCONH}_{2}\right)$ is creating cancer chemical which produced in certain foods due to heating process at high temperature and low humidity conditions. Due to the hazardous effect of acrylamide, in this study, the effect of frying temperature $\left(180\right.$ and $200^{\circ} \mathrm{C}$ ) and frying time (4 and 6 minutes) on the acrylamide levels has been discussed in fried beef burger using full factorial design of experiment (DOE). Produced acrylamide in each sample was analyzed by GC-FID system in the presence of internal standard. Between investigated parameters, results from DOE reveal that frying temperature has the higher impact on the produced acrylamide.
\end{abstract}

Keywords: Acrylamide, Frying temperature, Frying time, Full factorial design

DOI:10.3329/cerb.v15i1.7610

\section{Introduction}

Acrylamide, a potential genetic and reproductive toxin with mutagenic and carcinogenic properties in experimental mammalians in both in vitro and in vivo study, has been found in a large range of fried and baked carbohydrate-rich foods, which has attracted worldwide concern. Acrylamide has been classified as a probable carcinogen by the International Agency for Research on Cancer [1]. It is formed through Maillard reaction (MR) in many starch-rich foods [2].

It has been claimed that dietary acrylamide does not constitute any risk to human health [3, 4]; however, considering the potential health hazard of this molecule and its wide distribution in the Western diet, numerous studies have been undertaken to provide reliable mitigation strategies to minimize its levels in processed products [3-5].

Acrylamide is mainly formed in food by a reaction between asparagine and reducing sugars [6]. The mechanisms of acrylamide formation were deeply investigated [7, 8], and the significant role of 3aminopropionamide as reaction intermediate was elucidated $[9,10]$. Effective factors on the acrylamide formation such as cooking period, frying temperature, humidity, and amount of precursors for Maillard reaction, have been reported previously $[1,4,6,10]$. Since food products containing meat are also prone to acrylamide production, the aim of the current study was to investigate the effect of two important parameters (frying temperature and frying time) on the amount of produced acrylamide in fried beef burger.

\footnotetext{
*Corresponding author; Email: krezaee@ut.ac.ir

** Corresponding author; Email: r.abedini@modares.ac.ir
}

\section{Full factorial design of experiments (FUFE)}

When designing the basic experiment, the unknown response function is, in principle, approximated by a polynomial of the corresponding degree where regression coefficients are estimated on the basis of experimental results [11]. A linear mathematical model is considered in the first phase of a research. Defining the first order regression model is the first phase of study objectives at obtaining the interpolation model or function. The knowledge of which facilitates estimating response values in different points of the studied factorial space. In defining a linear model, coefficient $b_{0}$ and all linear regression coefficients are calculated Equation 1 [12].

$$
R=b_{0}+\sum_{i}^{k} b_{i} X_{i}+\sum_{i}^{k} b_{i j} X_{i} X_{j}
$$

where, $R$ is response value; $b_{i}$ are linear regression coefficients; $b_{i j}$ are regression coefficients of double factor interactions.

Accuracy and confidence of the obtained estimates for regression coefficients depend on the used design of experiments. Choice of the design of experiments has to do with determining the number of experimental points-trials and such a distribution of those points in a factorial space that facilitates obtaining the necessary information with a minimal number of design points-trials [13]. When selecting the design of experiments a design matrix or a standard type table is constructed where all conditions of doing design points that are part of the chosen design are defined. Mostly, in a design matrix, rows correspond to different design points-trials and columns to individual

(C)Bangladesh Uni. of Engg. E Tech. 
factors [13]. Obtaining a linear model has to do with performing full factorial experiment-FUFE, which is a definite part of FUFE. FUFE is the experiment where all possible combinations of levels of factors are realized and experimental results are processed by applying statistical analysis. The number of FUFE design points-trials is $2^{k}$ where $k$ is equal to the number of factors and factors are varied on two levels in FUFE [13].

\section{Materials and methods}

\subsection{Materials}

Hexane, potassium hexacyanoferrat (II) trihydrate (Carrez I), zinc sulfate heptahydrate (Carrez II), sodium sulfate, bromic acid, brome, sodium thiosulfate, sodium chloride, calcium chloride, ethylacetate, and acrylamide standard (99\%) from Merck chemical company (Darmstadt, Germany) were used. Potassium bromide and valeric acid were supplied from Acros Organic (Paris, France) and Sigma-Aldrich (St. Louis, MO) respectively.

\subsection{Sample preparation and analysis}

Sunflower oil (containing $0.01 \%$ TBHQ anti oxidant, $0.01 \%$ citric acid, $16 \%$ mono unsaturated fatty acid, $72 \%$ poly unsaturated fatty acid, and $12 \%$ saturated fatty acid) was used in this study. Fried beef burgers were maintained in the environment for 30 min and then they were distorted into powder form by means of mulinex blender. The powder was kept in refrigerator at $4^{\circ} \mathrm{C}$ temperature. Then, $30 \mathrm{~g}$ of powder was added to $70 \mathrm{ml}$ of hexane for defating. The mixture was stirred by a magnetic stirrer at $700 \mathrm{rpm}$. The yellow liquid of fat which produced due to stirring was separated manually and then, the sample was dried with nitrogen. Following that, $100 \mathrm{ml}$ of distilled water was added to the sample and the mixture was stirred by a magnetic stirrer for 1 hour at $700 \mathrm{rpm}$. During this stage, acrylamide was extracted into water phase.

Then the sample was centrifuged for $15 \mathrm{~min}$ at 4000 rpm. The upper transparent phase was moved to a balloon and increased to $100 \mathrm{ml}$ by addition of distilled water. For sedimentation of protein, $5 \mathrm{ml}$ Carrez I (150 g potassium hexacyanoferrat (II) trihydrate per liter) and $5 \mathrm{ml}$ Carrez II (300 g zinc sulfate heptahydrate per liter) were added to the solution and stirred at $500 \mathrm{rpm}$.

After protein sedimentation, the rest of the solution was centrifuged at $4000 \mathrm{rmp}$ for $15 \mathrm{~min}$. transparent phase was transferred to a container.

After acrylamide extraction by water, bromination process was carried out. At first $7.5 \mathrm{~g}$ of $\mathrm{KBr}$ and 0.5 $\mathrm{ml}$ of $\mathrm{HBr}$ were added to the sample and the mixture was shaken, then $2.5 \mathrm{ml}$ of saturated $\mathrm{Br}$ was added and stirred manually. The mixture was maintained in a dark place and $0^{\circ} \mathrm{C}$ environment for 90 minutes. These conditions are essential for bromination reaction. The sample was then transferred out from refrigerator and maintain until the temperature reached to the room temperature. After bromination, one molar solution of thiosulfate was instilled to the solution for $\mathrm{Br}$ neutralization. Instilling was continuing until the solution gets transparent. Finally, $4 \mathrm{~g}$ of $\mathrm{NaCl}$ was added to the mixture and stirred manually in order to enhance the extraction of ethyl acetate.

After derivitisation stage, the sample was transferred to a decanter and $20 \mathrm{cc}$ of ethylacetat and $5 \mathrm{cc}$ hexane were added and stirred for 10 minutes. Then 3 $\mathrm{g}$ of sodium sulfate was added to the mixture while it was stirring on the magnetic stirrer. Finally, this mixture was centrifuged and transparent phase was separated.

Finally the solution was filtered and the acrylamide was analyzed by a GC-FID system in the presence of valeric acid as an internal standard. Features of GCFID was capillary separating column of GC (PEG 10, Packed, 2 meter length and $=0.46 \mathrm{~cm} \mathrm{dm}$ ), FID detector was containing hydrogen and air at the speed of 33 and $330 \mathrm{ml} / \mathrm{min}$ respectively. Nitrogen by $99.999 \%$ purity at the rate of $30 \mathrm{ml} / \mathrm{min}$ was used as vector gas. Temperatures of injection chamber and detector were both $180^{\circ} \mathrm{C}$. The volume of injection was 1 ?. The temperature of the oven was scheduled as following: first at $85^{\circ} \mathrm{C}$ for one hour at the speed of 9 degrees in minutes, then the temperature increased up to $100^{\circ} \mathrm{C}$ at the speed of 10 degrees in minutes and finally the temperature enhanced up to $160^{\circ} \mathrm{C}$ at the speed of 20 degrees in minutes and the sample remained in that temperature for $5 \mathrm{~min}$.

\subsection{Experimental design}

The two important operating parameters that affect on production acrylamide in fried beef burger were frying temperature and the frying time. The effect of these variables on the target was evaluated using a twolevel factorial design. Table 1 presents the variables of interest and their real values at the levels set in the design. Each numeric variables was set to at a high $(+1)$ and a low $(-1)$ level. In the design matrix, the effects of two variables on produced acrylamide were evaluated. The runs were randomized for statistical purposes. Then, the data from run 1 to run 4 were computed and plotted using Design Expert 8 software.

\section{Results and Discussion}

\subsection{Half-normal probability plot}

Quantitatively, the estimated effects of a given main effect or interaction and its rank relative to other main 
Table 1: Design layout and experimental results of $2^{2}$ full factorial

\begin{tabular}{rrrrrrr}
\hline Std & Run & \multicolumn{2}{c}{ A: Temp. $\left({ }^{\circ} \mathrm{C}\right)$} & \multicolumn{2}{c}{ B: Time (min) } & R: Acrylamide (ppb) \\
& & Uncoded & Coded & Uncoded & Coded & \\
\hline 1 & 3 & 180 & -1 & 4 & -1 & 40 \\
2 & 2 & 200 & 1 & 4 & -1 & 76 \\
3 & 1 & 180 & -1 & 6 & 1 & 34 \\
4 & 4 & 200 & 1 & 6 & 1 & 85 \\
\hline
\end{tabular}

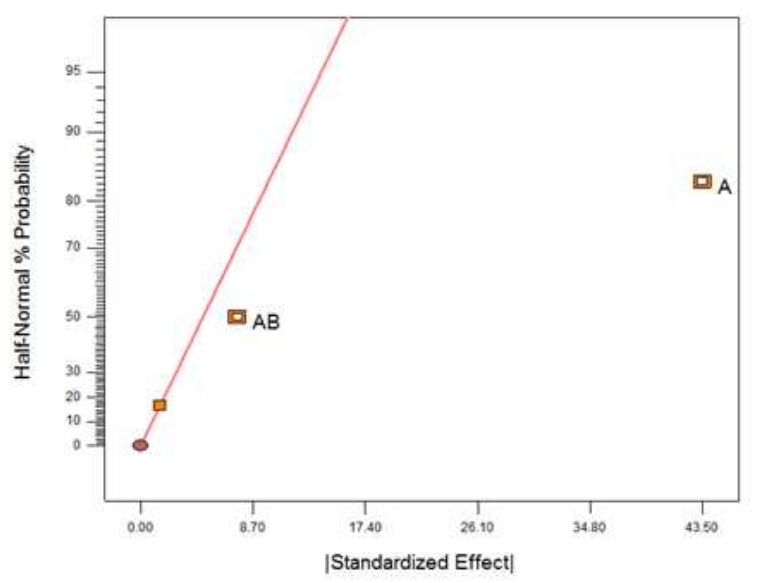

Figure 1: Half-normal probability plot for produced acrylamide

effects and interactions is given via least squares estimation (that is, forming effect estimates that minimize the sum of the squared differences between raw data and the fitted values from such estimates). Having such estimates in hand, one could then construct a list of the main effects and interactions ordered by the effect magnitude. The half-normal probability plot is a graphical tool that uses these ordered estimated effects to help assess which factors are important and which are unimportant. Figure 1 is a normal probability plot of the effects. All the effects that lie along the line were negligible, whereas larger ones are far from the line. Hence, the main effects including the frying temperature $(A)$, and interaction between temperature and time $(\mathrm{AB})$, effect significantly influenced the acrylamide production within the levels and conditions tested. Model hierarchy maintains the relationships between the main and interaction effects.

\subsection{Normal probability plot of residuals}

The normality of the data can be checked by plotting a normal probability plot of the residuals. If the data points on the plot fall fairly close to the straight line, then the data are normally distributed [14]. The normal probability plot of the produced acrylamide was shown in Figure 2. It can be seen that the data points were fairly close to the straight line and it indicates that the experiments come from a normally distributed population.

\subsection{Data analysis}

The data analysis was performed using DesignExpert Version 8 statistical software. The fitted mod-

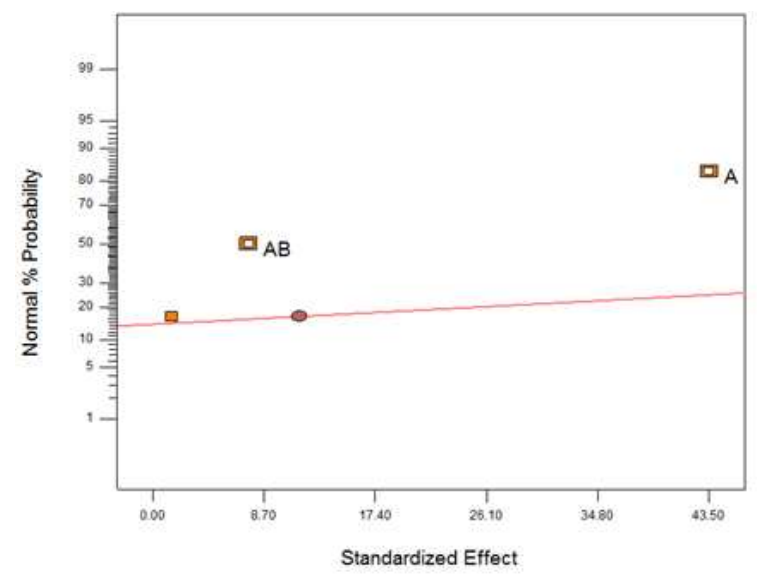

Figure 2: Normal probability plots of residuals for produced acrylamide

els were assessed with the coefficient of determination, $R^{2}$. A concern with this statistic is that it always increases as terms are added to the model, even though the added terms are often not significant. Consequently, this statistic is usually smaller for the refined model in comparison with corresponding full model. To negate this drawback, the adjusted coefficient of determination, $R^{2}-\mathrm{Adj}$; is used. This statistic is adjusted to the size of the model, more specifically, the number of factors. The addition of non significant terms to the model can usually decrease the $R^{2}$ Adj: value [15].

\subsection{ANOVA analysis}

In order to ensure a good model, test for significance of the regression model and test for significance on individual model coefficients must be performed. An ANOVA table is commonly used to summarize the tests that were performed. Table 2 shows the ANOVA table for produced acrylamide as a response. The $\mathrm{R}$-squared calculated is 0.998 , reasonably close to 1 , which is acceptable. It implies that about 0.996 of the variability in the data is explained by the model. Adequate precision compares the range of the predicted values at the design points to the average prediction error. The ratios greater than 4 indicate adequate model discrimination. In this case, the value is well above 4. Also the F-statistics (P-value $<0.05$ ) for the produced acrylamide as a response indicated that there was an adequate goodness-of-fit. Also plots of the residuals in Figure 3 revealed that they have no obvious pattern and unusual structure. They also show equal scatter above and below the $\mathrm{x}$-axis. This implies that the model proposed is adequate and there is no reason to suspect any violation.

In according to this study, the ranking is as follows: $\mathrm{A}>\mathrm{AB}$ for produced acrylamide in beef burger.

After the test, factors $\mathrm{A}$ and interaction $\mathrm{AB}$ exhibit statistically significant effects on the produced acry- 


\begin{tabular}{|c|c|c|c|c|c|}
\hline Source & $\sum$ Square & $\mathrm{dF}$ & in Square & $\overline{\text { F-Value }}$ & $\begin{array}{l}\text { P-Value } \\
\text { Prob }>\text { F }\end{array}$ \\
\hline Model & 1948.5 & 2 & 974.14 & 433.2 & 0.034 Significant \\
\hline A: Temperature & 1892.25 & 1 & 1892.26 & 841.51 & 0.0125 \\
\hline $\mathrm{AB}$ & 1350.56 & 1 & 56.36 & 388.34 & 0.0412 \\
\hline Residual & 2.25 & 1 & 2.25 & & \\
\hline Cor Total & 1950.75 & 3 & & & \\
\hline
\end{tabular}

Adeq Precision $=39.26$

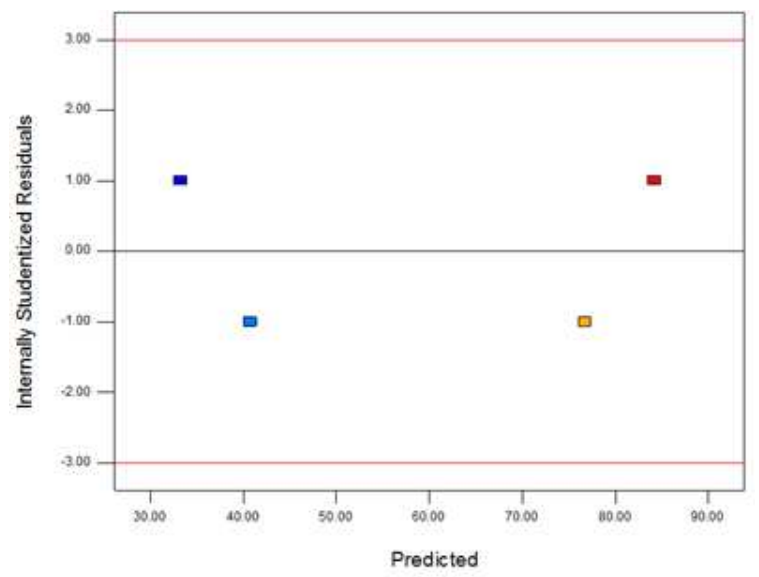

Figure 3: Plot of residual vs. predicted response for produced acrylamide

lamide. From a combination of estimates for the process variables and the ANOVA results, a polynomial model with statistical significance can be generated. This model, quantitatively elucidating the effects of process variables with statistical significance, is presented in terms of codec value as follows:

$$
R=58.75+21.75 \times A+3.75 \times A \times B
$$

Figure 4 revels the correlation between experimental data and those which predicted by model. It was observed that there is acceptable qualitative and quantitative conformity between predicted data by model results and experimental data.

\subsection{Main and interaction effects}

The effect of each factor was statistically significant at $\mathrm{P}<0.05$ [16]. For acrylamide production, the main effects $\mathrm{A}$ and the main interaction $\mathrm{AB}$ are of higher statistical significance. Based on P-value statistically insignificant factors were discarded.

Figure 5 shows the main effect for the frying temperature which has a significant effect on acrylamide production. The sign of the main effect indicates the directions of the effect. It can be seen from Figure 5 that the effect of frying temperature was characterized by a greater degree of departure and also had a negative effect on the response. Figure 6 reveals the interaction between frying temperature and time.

Contour plots for the significant factors frying temperature and frying time was shown in Figure 7. The

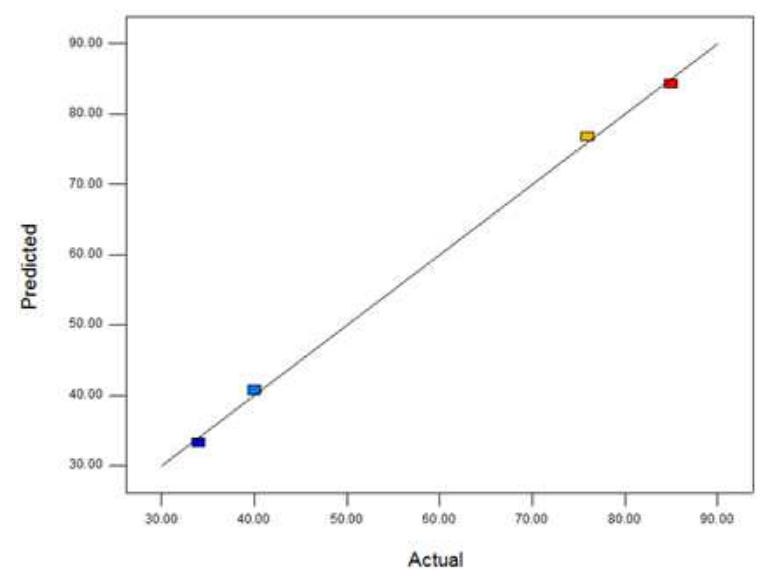

Figure 4: Correlation between experimental and predicted values of produced acrylamide responses using model

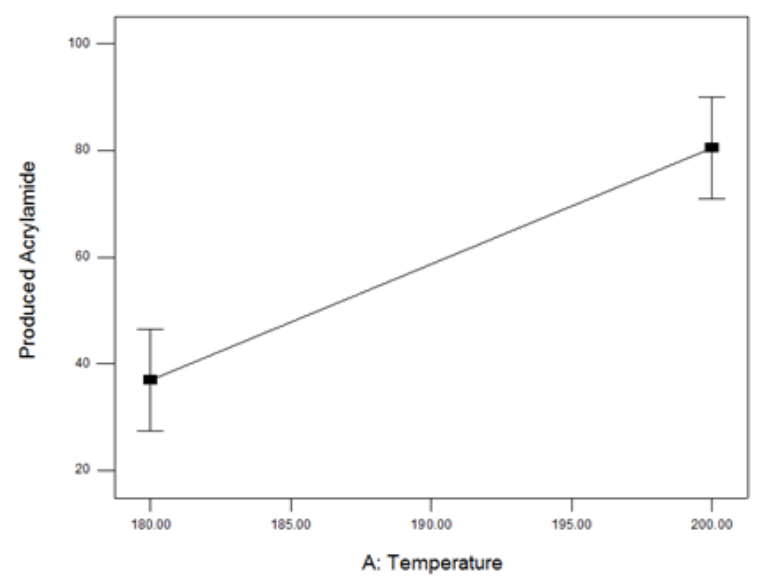

Figure 5: Main effect plot for frying temperature

interaction plots for produced acrylamide showed that interaction of temperature and time played major role and also these two factors interacted strongly with each other indicating predominant influence production of acrylamide. Surface response contour plots of the model represented by model could be constructed by varying frying temperature (A) and frying time (B). Figure 7 shows that with increasing temperature and time, rate of acrylamide production increases. Each of these two factors has negative impact on production levels of acrylamide. Countor graph shows that, the use of low frying temperature and frying time can lead to less production of acrylamide.

\section{Conclusion}

Effects of frying temperature and frying time as operational parameters were investigated on produced acrylamide in beef burger. Analysis of results carried out by using two-level full factorial design of experiment (FUFD) to avoid the traditional one-factor-at-atime experiments and the model developed by FUFD 


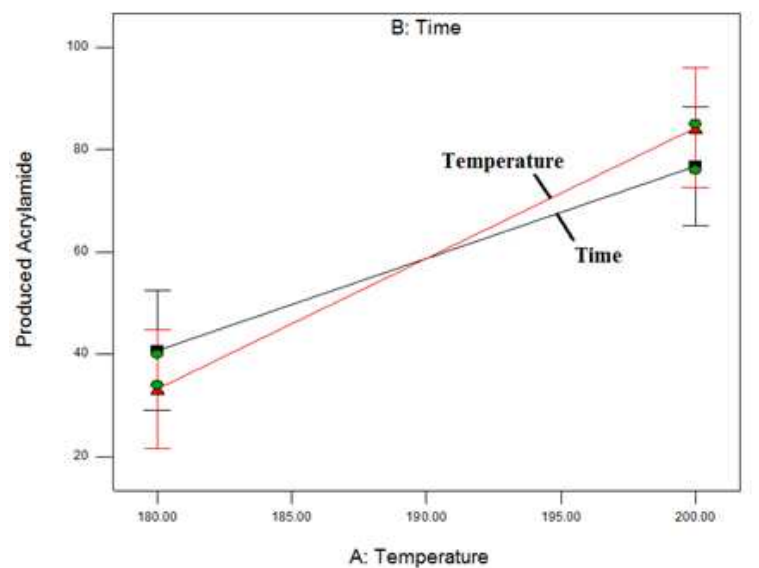

Figure 6: Interaction effect of factors

resulted in improved response, reduced process variability and closer confirmation of response to targeted requirements. Common statistical tools such as analysis of variance (ANOVA) and F-test were used to define the most important process variables affecting the production of acrylamde in beef burger. The use of FUFD allowed for identification of the most significant parameters under tested conditions. Main effects like frying temperature $(\mathrm{A})$ and the interactions $\mathrm{AB}$ were significant in acrylamide production. ANOVA reveal that between main factors, frying temperature due to has a highest F-Value show the most affect on acrylamide production.

\section{References}

[1] Carrieri G, De Bonis M, Pacella C, Pucciarelli A and Ruocco G, Modeling and validation of local acrylamide formation in a model food during frying, Journal of Food Engineering, 2009. 95(1):pp. 90-98. doi:10.1016/j.jfoodeng.2009.04.017

[2] Tareke E, Rydberg P, Karlsson P, Eriksson S and T "ornqvist M, Analysis of acrylamide, a carcinogen formed in heated foodstuffs, Journal of Agricultural and Food Chemistry, 2002. 50(17):pp. 4998-5006. doi:10.1021/jf020302f

[3] Jung MY, Choi DS and Ju JW, A novel technique for limitation of acrylamide formation in fried and baked corn chips and in French fries, Journal of Food Science, 2003. 68(4):pp. 12871290. doi:10.1111/j.1365-2621.2003.tb09641.x

[4] Carrieri G, Anese M, Quarta B, De Bonis MV and Ruocco $\mathrm{G}$, Evaluation of acrylamide formation in potatoes during deep-frying: The effect of operation and configuration, Journal of Food Engineering, 2010. 98(2):pp. 141-149. doi:10.1016/j.jfoodeng.2009.12.011

[5] Biedermann-Brem S, Noti A, Grob K, Imhof D, Bazzocco D and Pfefferle A, How much reducing sugar may potatoes contain to avoid excessive acrylamide formation during roasting and baking?, European Food Research and Technology, 2003. 217(5):pp. 369-373

[6] Pedreschi F, Kaack K, Granby K and Troncoso E, Acrylamide reduction under different pre-treatments in French fries, Journal of food engineering, 2007. 79(4):pp. 1287-1294. doi:10.1016/j.jfoodeng.2006.04.014

[7] Mottram D, Wedzicha B and Dodson A, Acrylamide is formed in the Maillard reaction, Nature, 2002. 419(6906):pp. 448449
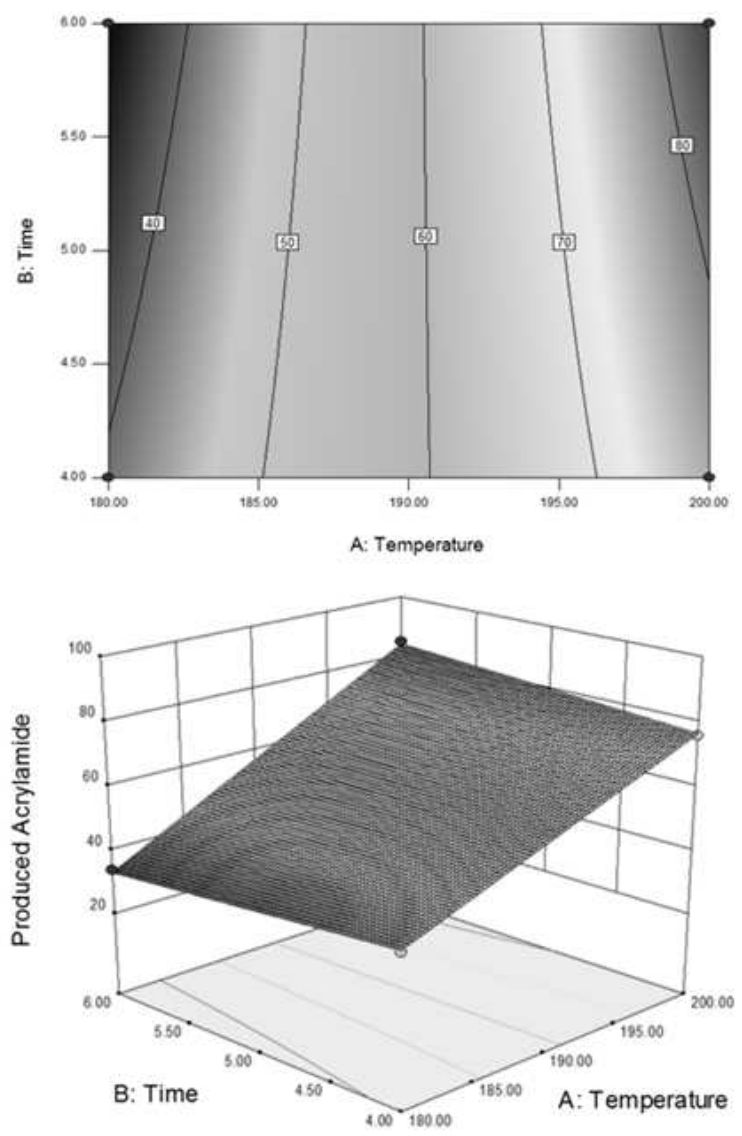

Figure 7: Contour graph of produced acrylamide respect to frying temperature and frying time, (b): 3-dimensions plot of level of produced acrylamide respect to the frying temperature and frying time

[8] Gokmen V, Palazoglu TK and Senyuva HZ, Relation between the acrylamide formation and time-temperature history of surface and core regions of French fries, Journal of food engineering, 2006. 77(4):pp. 972-976. doi:10.1016/j.jfoodeng.2005.08.030

[9] Wwisshaar R and Gutsche B, Formation of acrylamide in heated potato products-model experiments pointing to asparagine as precursor, Deutsche Lebensmittel-Rundschau, 2002. 98(11):pp. 397-400

[10] Franke K, Strijowski U and Reimerdes EH, Kinetics of acrylamide formation in potato powder, Journal of Food Engineering, 2009. 90(1):pp. 135-140. doi:10.1016/j.jfoodeng.2008.06.015

[11] Yamauchi A, Tanimoto J and Hagishima A, An analysis of network reciprocity in Prisoner's Dilemma games using Full Factorial Designs of Experiment, Biosystems, 2010. 103:pp. 85-92

[12] Meski S, Ziani S, Khireddine H and Zaidi SBS, Factorial design analysis for sorption of zinc on hydroxyapatite, Journal of hazardous materials, 2010. 186:pp. 1007-1017

[13] Porto CS, Porto TS, Nascimento KS, Teixeira EH, Cavada BS, Lima-Filho JL and Porto ALF, Partition of Lectin from Canavalia grandiflora Benth in aqueous two-phase systems using factorial design, Biochemical Engineering Journal, 2011. 53:pp. 165-171. doi:10.1016/j.bej .2010.10.006

[14] J A, Design of Experiments for Engineers and Scientists, Butterworth- Heinemann, New York, 2003

[15] Rahmanian B, Pakizeh M, Mansoori SAA and Abedini R, Application of experimental design approach and artificial neural network (ANN) for the determina- 
tion of potential micellar enhanced ultrafiltration process, Journal of Hazardous Materials, 2011. 187:pp. 67-74. doi:10.1016/j.jhazmat.2010.11.135

[16] Abdel NT, Hegazy AK, El-Chaghaby GA and Lima EC, Factorial experimental design for biosorption of iron and zinc using Typha domingensis phytomass, Desalination, 2009. 249:pp. 343-347. doi:10.1016/j.desal.2009.02.065 